\title{
NANOCOMPÓSITOS ELETROATIVOS DE POLI-O-METOXIANILINA E POLISSACARÍDEOS NATURAIS
}

\author{
Carla Eiras, Ionara Nayana Gomes Passos, Ana Cristina Facundo de Brito e José Ribeiro dos Santos Júnior
}

Departamento de Química, Centro de Ciências Naturais, Universidade Federal do Piauí, 64049-550 Teresina - PI, Brasil

Valtencir Zucolotto* e Osvaldo N. Oliveira Jr.

Instituto de Física de São Carlos, Universidade de São Paulo, CP 369, 13560-970 São Carlos - SP, Brasil

Igor L. Kitagawa e Carlos J. L. Constantino

Departamento de Física Química e Biologia, Faculdade de Ciências e Tecnologia, Universidade Estadual Paulista, CP 467, 19060-900 Presidente Prudente - SP, Brasil

Helder Nunes da Cunha

Departamento de Física, Centro de Ciências Naturais, Universidade Federal do Piauí, 64049-550 Teresina - PI, Brasil

Recebido em 27/6/06; aceito em 8/12/06; publicado na web em 30/7/07

\begin{abstract}
ELECTROACTIVE NANOCOMPOSITES MADE OF POLY( $O$-METHOXYANILINE) AND NATURAL POLYSACCHARIDES. In this work we take advantage of the polyelectrolyte character of some Brazilian native gums to fabricate electrically conductive, nanostructured films. The gums Sterculia urens, (caraia), Sterculia striata (chicha) or Anadenanthera macrocarpa Benth were assembled in conjunction with poly(o-methoxyaniline) (POMA) in the form of layered nanostructured films using the layer-bylayer (LbL) technique. All the LbL films displayed a well-defined electroactivity, as confirmed via cyclic voltammetry. In comparison to POMA LbL films fabricated with conventional polyelectrolytes (viz. poly(vinyl sulfonic acid)-PVS), the presence of the gums in the LbL films increased remarkably the electrochemical stability of the films.
\end{abstract}

Keywords: natural gums; poly-o-methoxyaniline; layer-by-layer films.

\section{INTRODUÇÃO}

Polissacarídeos naturais têm sido amplamente investigados nos últimos anos em relação às suas características físico-químicas e aplicações. Algumas de suas propriedades são biodegrabilidade, abundância na natureza e versatilidade de aplicações em engenharia, biotecnologia e medicina, além de serem geralmente atóxicos. A formação de filmes finos de polissacarídeos sobre superfícies sólidas pode gerar nova aplicação em sensores ou biossensores. Filmes de quitosana ${ }^{1}$, galactomananas ${ }^{2}$ e de carboximetilcelulose ${ }^{3}$ vêm sendo usados para fins variados. As gomas naturais são polissacarídeos obtidos de exsudatos de tronco de árvore, sementes, algas ou por fermentação microbiológica. Esses materiais podem ser hidratados em água fria ou quente formando dispersões coloidais, soluções altamente viscosas ou até mesmo géis. Os exsudatos são obtidos do tronco de árvores como resposta do mecanismo de proteção da planta ${ }^{4,5}$. A liberação do exsudato ocorre de forma espontânea ou exploratória, como é o caso do látex da seringueira. Condições climáticas desfavoráveis e solos pobres também estimulam esta produção ${ }^{4,5}$. No entanto, a formação de exsudato pode também ser causada pela indução de agentes químicos, como o óxido de etileno e derivados de ácido benzóico.

A árvore angico (Anadenanthera macrocarpa Benth) pertence à família das leguminosas e exsuda uma goma com as mesmas características das gomas arábica (Acacia senegal) e do cajueiro (Anacardium occidentale). A composição da goma do angico, um heteropolissacarídeo, contém arabinose $(67,8 \%)$, galactose $(24,1 \%)$, ácido urônico $(5,9 \%)$ e traços de ramnose, além de apresentar baixa viscosidade, semelhante às gomas do cajueiro e arábica ${ }^{6,7}$. A goma extraída da árvore do chichá (Strerculia striata) é encontra-

*e-mail: zuco@if.sc.usp.br da no norte e no nordeste do Brasil. As gomas obtidas das árvores da família das Sterculias são conhecidas por apresentarem teores elevados de ácidos e alta viscosidade, além de serem capazes de formar géis em água. Um exemplo da goma que apresenta estas características é a exsudada pela árvore caraia (Sterculia urens), nativa da Índia, utilizada principalmente como agente espessante e gelificante. A goma do chichá contém galactose, ramnose e xilose e elevado percentual de ácido urônico $(42,2 \%)$, além de ser parcialmente acetilada $(10,7 \%)^{8}$. A adição de sais às soluções de goma e a presença do grupo acetil influenciam diretamente na viscosidade da mesma9.

A formação de filmes do tipo automontados envolve a deposição de camadas através de interações eletrostáticas entre soluções de cargas contrárias envolvendo diferentes processos de adsorção ${ }^{10}$. A adsorção resultante da interação eletrostática entre o material suporte (polímero sintético) e o grupamento $\mathrm{COO}^{-}$da goma parece solucionar o problema de elevada hidrofilicidade dos filmes formados só por gomas e proporciona uma nova perspectiva de aplicações para estas blendas.

A POMA é derivada da polianilina (PANi), cuja diferença estrutural é a presença de um grupo metoxi $\left(-\mathrm{OCH}_{3}\right)$ na posição orto dos anéis de carbono nos meros na PANi. Apesar dessa diferença nos meros, a POMA apresenta propriedades físicas bastante semelhantes às da PANi. Porém, sua solubilidade é maior que a da PANi, o que se deve a dois efeitos ${ }^{11}$ promovidos pela presença do grupo metoxi: torção induzida na cadeia polimérica e aumento da polaridade da mesma ${ }^{12,13}$. Assim como a PANi, a POMA pode ser obtida em diferentes estados de oxidação: leucoesmeraldina, esmeraldina e pernigranilina, sendo que o estado de oxidação esmeraldina pode ser encontrado nas formas de base (não condutor elétrico) ou de sal (condutor elétrico). A conversão de base para sal de esmeraldina pode ser obtida via protonação em meio ácido, daí tais estados 
serem chamados também de não dopado e dopado, respectivamente. Também para a POMA, a forma esmeraldina é a mais comumente utilizada. A POMA vem sendo bastante estudada ${ }^{14-17}$ na forma obtida via síntese química (pó) ou via eletroquímica (filme) ${ }^{18-20} \mathrm{com}$ a finalidade das mais diversas aplicações, tais como em conjunto com DNA para fins biomédicos ${ }^{21}$ ou na fabricação de filmes automontados $^{22-25}$. Sousa e co-autores ${ }^{22}$ estudaram os processos de adsorção na preparação de filmes automontados de POMA com poli(vinil sulfonato de sódio) (PVS) empregando um método estatístico para tratar as propriedades morfológicas dos filmes.

Neste trabalho foram preparados filmes finos multicamadas usando as gomas do chichá, do angico e da caraia em conjunto com o polímero sintético e condutor poli-o-metoxianilina (POMA), usando a técnica de automontagem ${ }^{10}$. Os filmes automontados de POMAgomas foram caracterizados pelas técnicas de espectroscopia na região do UV-VIS, micro-espectroscopia Raman e pela técnica eletroquímica de voltametria cíclica.

\section{PARTE EXPERIMENTAL}

As gomas do chichá e angico foram coletadas no estado do Piauí, em agosto de 2004, e purificadas na forma de sal de sódio como descrito na literatura ${ }^{25}$. Após o processo de purificação, uma massa de $0,5 \mathrm{~g}$ de cada goma foi dissolvida em $100 \mathrm{~mL}$ de água por $24 \mathrm{~h}$, sendo as soluções filtradas em funil de placa sinterizada, adicionado-se $0,25 \mathrm{~mL}$ de álcool para ajudar na evaporação do solvente. A goma caraia é da família das Sterculias, assim como o chichá, e é originária da Índia, sendo adquirida comercialmente (Synth). A solução de caraia também foi preparada segundo o procedimento utilizado para as gomas chichá e angico. Todas estas soluções apresentaram $\mathrm{pH}$ em torno de 3,0 e foram utilizadas diretamente na fabricação dos filmes automontados. A POMA foi obtida por via química na forma de base esmeraldina, isolante, de acordo com a rota descrita por Mattoso e colaboradores ${ }^{26}$. A Figura 1 mostra o oligômero da POMA nas formas de base e de sal de esmeraldina. Depois de sua preparação, a POMA (em pó) foi dissolvida em dimetilacetamida (DMAc) e colocada sob agitação por $12 \mathrm{~h}$. A solução de POMA/ DMAc foi filtrada, ajustando-se em seguida o $\mathrm{pH}$ da solução para 2,5 através da adição de solução de $\mathrm{HCl} 0,1 \mathrm{~mol} \mathrm{~L}^{-1}$. A solução aquosa de POMA foi diluída para a concentração de $1,2 \mathrm{~g} \mathrm{~L}^{-1}$ e mantida em pH 2,5. O método para a obtenção da POMA solúvel em água foi descrito de forma detalhada por Cheung e colaboradores ${ }^{27}$.

a)<smiles>CNc1ccc(Nc2ccc(N=C3C=CC(=Nc4ccc(C)cc4OC)C=C3OC)cc2OC)cc1OC</smiles>

b)

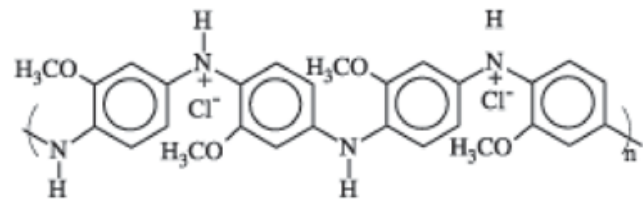

Figura 1. Estrutura química de um monômero da POMA na forma esmeraldina base (a) e sal (b)

A deposição das multicamadas foi feita manualmente através do seguinte procedimento: o substrato previamente limpo ${ }^{28}$ foi imerso na solução do polieletrólito catiônico (POMA) por um período de 5 min e então lavado em solução de $\mathrm{HCl}$ pH 2,5 para a remoção do material não-adsorvido; o sistema substrato/filme foi imerso em solução do polieletrólito aniônico (gomas) também por um período de 5 min e lavado em solução em pH 2,5. Depois da deposição de cada camada, o sistema substrato/filme foi seco sob fluxo de nitrogênio, obtendo-se uma bicamada de POMA-goma. O processo é repetido até se atingir o número desejado de bicamadas.

A formação das multicamadas foi monitorada em cada etapa de deposição por espectroscopia de absorção UV-VIS em filmes depositados sobre substratos de vidro BK7. Para análise dos espectros de UV-VIS foram preparados filmes utilizando as gomas angico, chichá e caraia e o poli(vinil-sulfonato de sódio) (PVS) como soluções aniônicas. Os filmes de POMA-PVS foram utilizados para comparação, pois nesse caso é possível analisar a POMA em um filme automontado na ausência das gomas. Os espectros Raman e as imagens ópticas dos filmes (obtidos em filmes automontados depositados sobre substratos de vidro recobertos com ouro) foram coletados através do espectrógrafo micro-Raman Renishaw, modelo in-Via com microscópio Leica acoplado ao espectrógrafo com lente de 50x e laser de $785 \mathrm{~nm}$ (NIR). Neste caso, foi utilizado ouro para se evitar o ruído de fundo proveniente do vidro. Ressaltamos que os filmes foram imersos em solução de $\mathrm{HCl} \mathrm{pH} 2,5$ previamente à realização das medidas. Os voltamogramas cíclicos foram obtidos com filmes automontados depositados sobre ITO (vidro recoberto com uma fina camada de óxido de estanho dopado com índio) usando um potenciostato/galvanostato Autolab PGSTAT30 e uma célula eletroquímica com volume total de $20 \mathrm{~mL}$ e tampa com encaixe para três eletrodos, além de uma entrada e uma saída para gás. $\mathrm{O}$ eletrodo de referência foi o eletrodo saturado de calomelano (ESC), uma placa de platina com área $1,0 \mathrm{~cm}^{2}$ foi usada como contra eletrodo e o eletrodo de trabalho foi o filme automontado depositado sobre ITO. Os experimentos foram realizados em solução de $\mathrm{HCl} 0,1 \mathrm{~mol} \mathrm{~L}^{-1}$ em sala climatizada à temperatura de $22^{\circ} \mathrm{C}$.

\section{RESULTADOS E DISCUSSÃO}

\section{Caracterização via absorção no UV-VIS}

Na Figura 2 são apresentados os espectros de UV-VIS para os filmes de POMA-caraia, POMA-chichá e POMA-angico, juntamente com o espectro do filme de POMA-PVS obtidos imediatamente após suas preparações. Os espectros mostram uma banda de absorção em $620 \mathrm{~nm}$ correspondente à transição eletrônica característica da POMA, uma vez que as gomas puras não absorvem nessa região. O espectro de UV-VIS observado nesta figura é característico da POMA no estado base esmeraldina, sugerindo que a interação com as gomas e com o PVS resulta em desdopagem da POMA, fato este semelhante ao que já havia sido relatado para um sistema similar ${ }^{29}$.

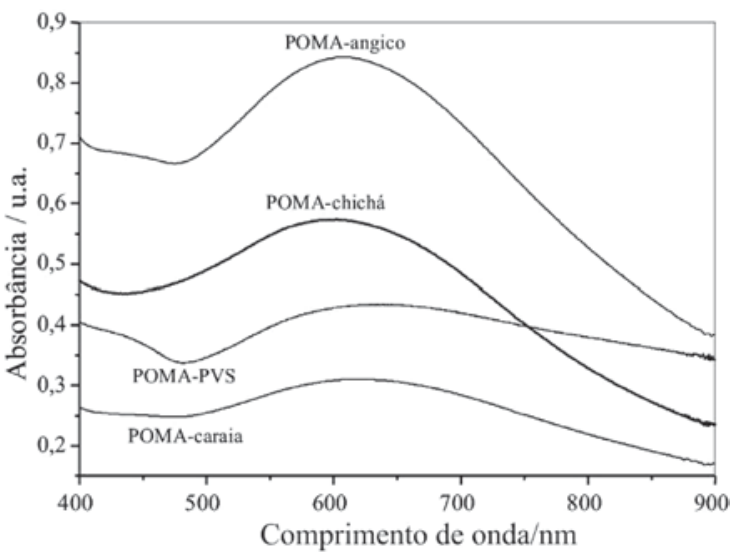

Figura 2. Espectro de absorção eletrônica na região do UV-VIS dos filmes automontados com 20 bicamadas de POMA-PVS, POMA-caraia, POMAchichá e POMA-angico 
O crescimento seqüencial dos filmes automontados em função das camadas é apresentado na Figura 3. A espessura dos filmes pode ser controlada pelo número de bicamadas depositadas e da goma utilizada. Um crescimento linear pode ser observado para todos os filmes, indicando que a mesma quantidade de material é adsorvida em cada etapa de deposição, respectivamente. Pode-se ainda observar a diferença de inclinação das curvas dependendo da goma utilizada, sendo que maiores inclinações podem estar relacionadas à maior afinidade entre o polímero, através dos $\mathrm{N}$ amina, e o grupamento - $\mathrm{OH}$ das gomas. Destacam-se nestes casos as gomas do chichá e do angico.

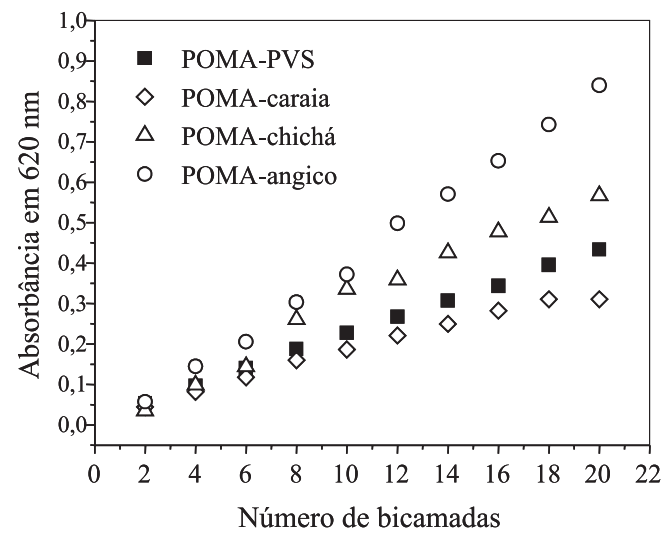

Figura 3. Aumento da absorbância em $620 \mathrm{~nm}$ em função do número de bicamadas dos filmes automontados de POMA-gomas e POMA-PVS

Caracterização vibracional e morfológica por microespectroscopia Raman

Uma caracterização preliminar via espectroscopia Raman foi realizada para a base e o sal de POMA esmeraldina, POMA-EB e POMA-ES, respectivamente, para que tais espectros servissem de referência para a posterior caracterização dos filmes automontados contendo POMA-gomas. A Figura 4 mostra os espectros Raman para a POMA-EB (pó oriundo da síntese) e POMA-ES (filme "casting" de POMA esmeraldina obtido da dissolução do pó da POMA-EB em DMAc e ajustado o $\mathrm{pH}=2,5$ ). Alguns picos dos estados dopado e não dopado da POMA esmeraldina despertam interesse por existirem em somente um dos estados, ou diminuírem drasticamente de intensidade. Os picos Raman característicos da POMA-EB são: 1207 e $1260 \mathrm{~cm}^{-1}$ atribuídos ao estiramento C-N (unidades benzênicas) e $1480 \mathrm{~cm}^{-1}$ atribuído ao estiramento $\mathrm{C}=\mathrm{N}$ (unidades quinóides). Alguns picos Raman característicos da POMA-ES são: $577 \mathrm{~cm}^{-1}$ atribuído à deformação da amina no plano, $1356 \mathrm{~cm}^{-1}$ atribuído ao cátion radical (estiramento C-N+) e 1597

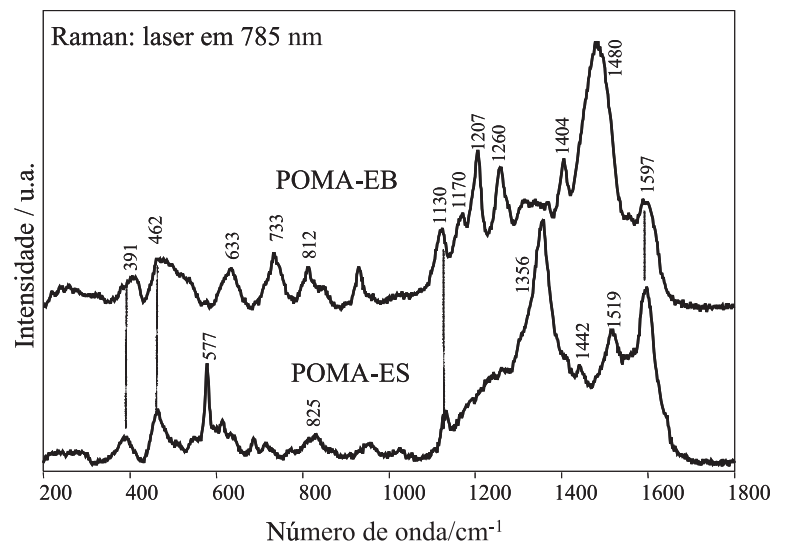

Figura 4. Espectros Raman da base (POMA-EB) e do sal (POMA-ES) de POMA esmeraldina $\mathrm{cm}^{-1}$ atribuído ao estiramento C-C do anel benzeno. A Tabela 1 traz os picos Raman da base e do sal de POMA esmeraldina, e suas respectivas atribuições, as quais são "atribuições tentativas" baseadas nas referências ${ }^{30-35}$ que se referem à PANi.

Tabela 1. Bandas Raman da base (POMA-EB) e do sal (POMAES) de POMA esmeraldina com suas respectivas atribuições

\begin{tabular}{|c|c|c|}
\hline POMA-EB $\mathrm{cm}^{-1}$ & POMA-ES $\mathrm{cm}^{-1}$ & Atribuições ${ }^{1,2}$ \\
\hline & 391 & $\begin{array}{l}\text { Deformação do anel } \\
\text { benzeno (no plano) }\end{array}$ \\
\hline & 462 & Torsão C-N-C (fora do plano) \\
\hline & 577 & Deformação da amina (no plano) \\
\hline 633 & & $\begin{array}{l}\text { Deformação do anel } \\
\text { benzeno (no plano) }\end{array}$ \\
\hline 733 & & $\begin{array}{l}\text { Deformação C-C do anel } \\
\text { benzeno (fora do plano) }\end{array}$ \\
\hline 812 & & $\begin{array}{l}\text { Deformação C-H do anel } \\
\text { quinóide (fora do plano) }\end{array}$ \\
\hline 1130 & 1130 & $\begin{array}{l}\text { Deformação C-H do anel } \\
\text { benzeno (no plano) }\end{array}$ \\
\hline 1170 & & $\begin{array}{l}\text { Deformação C-H do anel } \\
\text { quinóide (no plano) }\end{array}$ \\
\hline 1207 & & Estiramento $\mathrm{C}-\mathrm{N}$ \\
\hline \multirow[t]{2}{*}{1260} & & Estiramento C-N \\
\hline & 1356 & $\begin{array}{c}\text { Cátion radical } \\
\left(\mathrm{C}-\mathrm{N}^{+} \text {estiramento }\right)\end{array}$ \\
\hline \multirow[t]{2}{*}{1404} & & $\begin{array}{l}\text { Estiramento C-C do } \\
\text { anel quinóide }\end{array}$ \\
\hline & 1442 & $\begin{array}{l}\text { Deformação do anel } \\
\text { benzeno (no plano) }\end{array}$ \\
\hline 1480 & & Estiramento $\mathrm{C}=\mathrm{N}$ \\
\hline & 1519 & Deformação N-H(no plano) \\
\hline 1597 & 1597 & Estiramento $\mathrm{C}-\mathrm{C}$ do anel benzeno \\
\hline
\end{tabular}

1As atribuições "no plano" ou "fora do plano" referem-se ao plano do anel benzeno. "A atribuição "deformação" ("bending") referese à deformação angular. Se "scissoring" ou "rocking" (deformações no plano), "wagging" ou "twisting" (deformações fora do plano), não se pode precisar na literatura.

\section{Estudo da eletroatividade dos filmes contendo gomas naturais}

A caracterização eletroquímica dos filmes POMA-gomas foi realizada utilizando-se a técnica de voltametria cíclica em meio ácido com os filmes depositados sobre ITO. O filme automontado de POMA-PVS foi utilizado para comparação, conforme discutido anteriormente. Para as análises eletroquímicas, filmes de mesmo número de bicamadas e mesma área de substrato foram utilizados para o estudo da variação da corrente em função do potencial aplicado. Inicialmente, para verificar a influência da goma na resposta eletroquímica dos filmes, foi analisado um filme de POMA-caraia (50 bicamadas), em comparação com um filme de POMA-PVS, com o mesmo número de bicamadas. Uma característica importante dos filmes de POMA, quando automontados com as gomas, é a melhora acentuada em sua estabilidade em meio ácido quando comparado com POMA-PVS. A Figura 5 mostra respostas eletroquímicas dos filmes de POMA-PVS e POMA-caraia, ambos com 50 bicamadas. Realizando-se vários ciclos voltamétricos em solução ácida ( $\mathrm{HCl} 0,1 \mathrm{~mol} \mathrm{~L}^{-1}$ ), a POMA do filme POMA-PVS (Figura 5a) sofre um processo irreversível de degradação ${ }^{36}$, do qual advém um par redox intermediário, na região de $+0,3 \mathrm{~V}$ vs ESC, a partir do terceiro ciclo de varredura (como indicado pelas setas). 

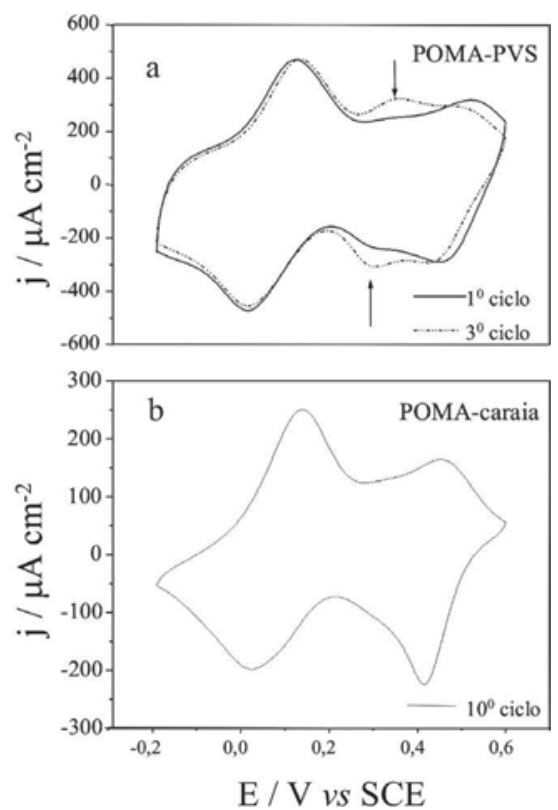

Figura 5. Voltamogramas de resposta de filmes automontados contendo 50 bicamadas de POMA-PVS (a); POMA-caraia (b). HCl 0,1 mol L-1 $50 \mathrm{mV} \mathrm{s}^{-1}$

Esta degradação, no entanto, não foi verificada no filme de POMAcaraia (Figura 5b), que mesmo após 10 ciclos voltamétricos em meio ácido não apresenta o par redox intermediário.

A resposta eletroquímica do filme de POMA-caraia na Figura 5b mostra dois pares redox característicos de um filme eletroativo de POMA, com os processos de oxidação-redução correspondentes às seguintes transições de estados: o primeiro processo (a partir de $-0,2 \mathrm{~V}$ $v s$ ESC) está relacionado com a conversão da base leucoesmeraldina (amarelo claro) à forma protonada da esmeraldina (verde) $(+0,12 \mathrm{~V} v s$ ESC); o segundo processo anódico corresponde à conversão da forma esmeraldina protonada para a estrutura pernigranilina totalmente oxidada (violeta) (com máximo em $+0,41 \mathrm{~V}$ vs ESC) ${ }^{36-39}$.

Como os filmes de POMA-chichá são mais homogêneos em termos morfológicos e crescem de forma mais efetiva adsorvendo mais material por bicamada depositada que os filmes automontados das outras gomas, realizamos um estudo eletroquímico detalhado desse sistema. A Figura 6a mostra a resposta eletroquímica para filmes de POMA-chichá com diferentes números de bicamadas: 2, 5, 10, 15 e 20. Os processos redox da POMA foram observados em todos os casos. É importante ressaltar que a utilização da goma é vantajosa do ponto de vista eletroquímico, em relação ao uso de PVS, uma vez que filmes de POMA-chichá com 10 bicamadas apresentam os processos redox bem definidos e altos valores de densidades de corrente. Uma possível explicação para este fato é a presença de maior quantidade de POMA, devido à adsorção ser mais eficiente quando a goma é utilizada como polieletrólito caracterizando interação entre os materiais depositados. Na Figura $6 b$ utilizou-se um filme de POMA-chichá com 20 bicamadas para estudar a eletroatividade em soluções de diferentes pHs. Em pH 1 o filme apresenta os processos redox do filme eletroativo de POMA-chichá. Em pH 2,5, no entanto, o voltamograma se altera, com apenas um pico de oxidação $(+0,31$ $\mathrm{V}$ vs ESC) na varredura direta, e seu pico de redução correspondente (+0,11 V vs ESC) na varredura inversa. Em pH 3,0 estes processos sofrem um deslocamento ainda maior e em pHs 4,0 e 5,0 nenhuma eletroatividade foi observada para o filme de POMA-chichá. Esse é um comportamento característico de POMA quando submetido a varreduras de potenciais (processo eletroquímico) na presença de ácidos, os quais realizam a protonação do polímero promovendo a

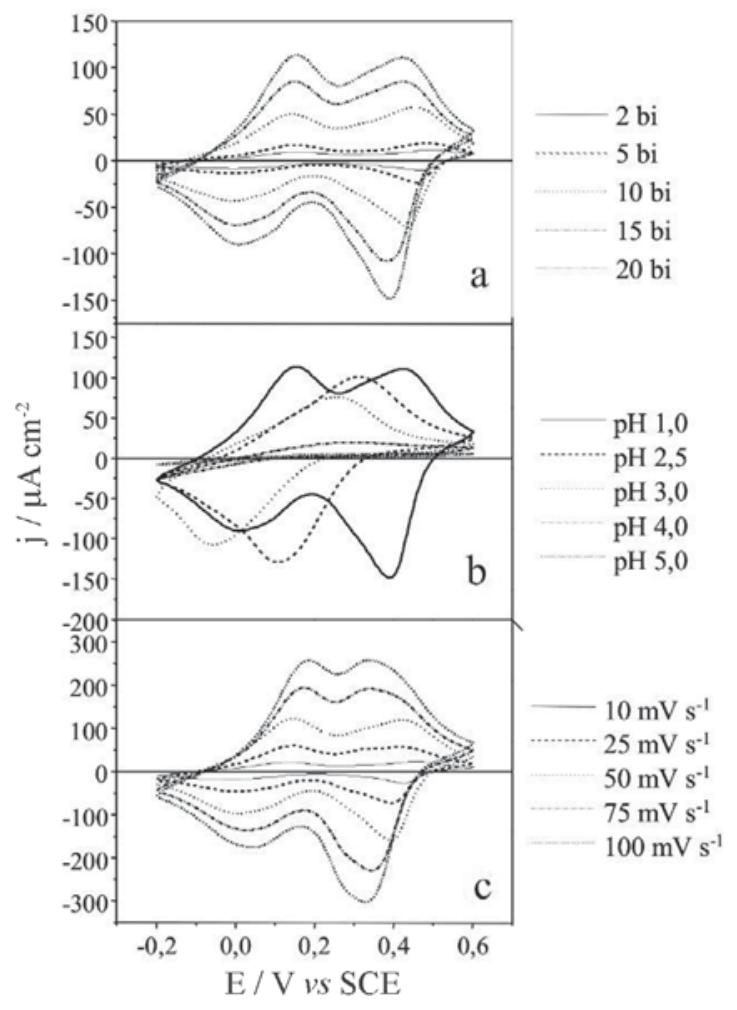

Figura 6. Voltamogramas cíclicos dos filmes automontados de POMA-chichá

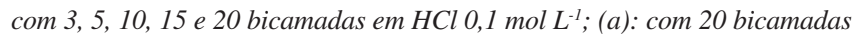
em solução de diferentes $\mathrm{pHs}(1,0 ; 2,5 ; 3,0 ; 4,0$ e 5,0); (b): com 20 bicamadas em $\mathrm{HCl} 0,1 \mathrm{~mol} \mathrm{~L}^{-1}$ e diferentes velocidades de varredura (c)

condutividade do filme. Variando-se o $\mathrm{pH}$ o filme de POMA muda o seu grau de protonação, perdendo atividade eletroquímica ${ }^{40}$. No entanto, ao ser ciclado novamente em pH 1,0 o filme de POMA-chichá volta a mostrar comportamento eletroativo. A Figura $6 \mathrm{c}$ mostra as respostas eletroquímicas do filme de POMA-chichá de 20 bicamadas em $\mathrm{HCl} 0,1 \mathrm{~mol} \mathrm{~L}^{-1}$ obtido com diferentes velocidades de varredura. Como pode ser verificado, para velocidades de varredura superiores a $50 \mathrm{mV} \mathrm{s}{ }^{-1}$ existe uma aproximação dos picos em relação ao voltamograma obtido nesta varredura. Essa aproximação é explicada pela perda de protonação da POMA, em função da entrada e saída dos íons no filme. O processo é semelhante à variação do $\mathrm{pH}$, quando não existem íons $\left(\mathrm{H}^{+}\right)$disponíveis no meio para efetuar a protonação, nos processos em que a velocidade de varredura é a variável e não o pH. Não existe tempo de difusão suficiente para equilibrar a entrada e saída dos íons e promover a adequada oxidação por protonação ${ }^{40}$.

\section{CONCLUSÕES}

Filmes automontados envolvendo diferentes polieletrólitos aniônicos (gomas e PVS) e o polímero condutor POMA como polieletrólito catiônico apresentaram diferenças tanto na quantidade de material adsorvido por bicamada produzida pela técnica de automontagem quanto na morfologia da superfície do filme em escala micrométrica. Neste contexto, dentre os filmes POMA-gomas destaca-se o POMA-chichá por sua uniformidade morfológica em escala micrométrica e alta adsorção de material por bicamada em relação aos demais. Por outro lado, independentemente dos filmes (POMA-gomas ou POMA-PVS), todos apresentaram uma uniformidade química mostrada pelos espectros Raman coletados em diferentes regiões dos filmes. A espectroscopia de absorção no UV- 
VIS mostrou um crescimento linear da absorbância destes filmes (acompanhado pela banda em $620 \mathrm{~nm}$ ) e que a dopagem da POMA é afetada pela presença dos polieletrólitos, os quais induzem uma desdopagem na POMA. Os espectros de Raman, porém, mostraram que os filmes podem ser novamente dopados após a preparação e assim permanecerem. A análise eletroquímica mostrou que as gomas conferem estabilidade eletroquímica à POMA em meio ácido durante ciclagens sucessivas quando comparaas com o PVS, além de manterem as propriedades eletrocrômicas da POMA.

\section{MATERIAL SUPLEMENTAR}

Encontra-se disponível gratuitamente em http://quimicanova. sbq.org.br, na forma de arquivo pdf.

\section{AGRADECIMENTOS}

Ao apoio financeiro da FAPEPI, FAPESP, CNPq e IMMP/MCT e ao LAPETRO-UFPI pelo apoio técnico.

\section{REFERÊNCIAS}

1. Huguenin, F.; Zucolotto, V.; Carvalho, A. J. F. .; Gonzalez, E. R.; Oliveira Jr, O. N.; Chem. Mat. 2005, 17, 6739.

2. Figueiró, S. D.; Góes, J. C.; Moreira, R. A.; Sombra, A. S. B.; Carbohydr Polym. 2004, 56, 313.

3. Fujimoto, J.; Reis, E. A. de O.; Petri, D. F. S.; Campana Filho, S. P.; Quim. Nova 2002, 25, 757.

4. Anderson, D. M. W.; Macdougall, F. F.; Food Hydrocolloids 1987, 1, 137.

5. Rosenthal, F. R. J.; Revista Química Industrial 1995, 24, 17.

6. Paula, R. C. M.; Budd, P. M.; Rodrigues, J. F.; Polym. Int. 1997, 44, 55.

7. Silva, A. G.; Rodrigues, J. F.; Paula, R. C. M.; Polímeros: Ciência e Tecnologia 1998, abr/jun, 34.

8. Brito, A. C. F.; Silva, D. A.; Paula, R. C. M.; Feitosa, J. P. A.; Polym. Int. 2004, 53, 1025.

9. Silva, D. A.; Paula, R. C. M.; Feitosa, J.; Brito, A. C. F.; Maciel, J. S.; Paula, H. C. B; Carbohydr. Polym. 2004, 58, 163.

10. Paterno, L. G.; Mattoso, L. H. C.; Oliveira Jr., O. N.; Quim. Nova 2001, 24, 228.

11. Gonçalves, D.; dos Santos Jr., D. S.; Mattoso, L. H. C.; Karasz, F. E.; Akcelrud, L.; Faria, R. M.; Synth. Met. 1997, 90, 5.

12. Wei, Y.; Focke, W. W.; Wnek, G. N.; Ray, A.; MacDiarmid, A. G.; J. Phys. Chem. 1989, 93, 495.

13. Leclerc, M.; Guay, J.; Dao, L. H.; Macromolecules 1989, 22, 649.

14. Marletta, A.; Piovesan, E.; Dantas, N. O.; de Souza, N. C.; Olivati, C. A.; Balogh, D. T.; Faria, R. M.; Oliveira Jr., O. N.; J. Appl. Phys. 2003, 94, 5592 .
15. de Souza, N. C.; Tese de Doutorado, Universidade de São Paulo, Brasil, 2002.

16. Mattoso, L. H. C.; Tese de Doutorado, Universidade de São Paulo, Brasil, 1993.

17. Huerta-Vilca, D.; Siefert, B.; Moraes, S. R.; Pantoja, M. F.; Motheo, A. J.; Mol. Cryst. Liq. Cryst. 2004, 415, 229.

18. Lobo, R. F. M.; Pereira-da-Silva, M A.; Raposo, M.; Faria, R. M.; Oliveira Jr., O. N.; Nanotechnology 2003, 14, 101.

19. Gazotti, W. A.; de Freitas, P. S.; Waldman, W. R.; de Paoli, M. A.; Synth. Met. 1999, 102,1207.

20. Komsiyska, L.; Tsacheva, T.; Tsakova, V.; Thin Solid Films 2005, 493, 88.

21. Dawn, A.; Nandi, A. K.; Macromolecules 2005, 24, 10067.

22. de Souza, N. C.; Silva, J. R.; di Thommazo, R.; Raposo, M.; Balogh, D. T.; Giacometti, J. A.; Oliveira Jr., O. N.; J. Phys. Chem. 2004, 108, 13599.

23. de Souza, N. C.; Silva, J. R.; Pereira-da-Silva, M. A.; Raposo, M.; Faria, R. M.; Giacometti, J. A.; Oliveira Jr., O. N.; J. Nanosci. Nanotechnol. 2004, 4, 548.

24. Raposo, M.; Oliveira Jr., O. N.; Langmuir 2002, 18, 6866.

25. Costa, S. M. O.; Rodrigues, J. P. A.; De Paula, R. C. M.; Polímeros: Ciência e Tecnologia 1996, 2,49 .

26. Mattoso, L. H. C.; MacDiarmind, A. G.; Epstein, A. J.; Synth. Met. 1994, $68,1$.

27. Cheung, J. H.; Stockton, W. B.; Rubner, M. F.; Macromolecules 1997, 30, 2712.

28. Kern W.; Semiconductor International 1994, 4, 94.

29. Piza, M. A.; Constantino C. J. L.; Venancio, E. C.; Mattoso L. H. C.; Polymer 2003, 44, 5663.

30. Cochet, M.; Louam, G.; Quillard, S.; Buisson, J. P.; Lefrant, S.; J. Raman Spectrosc. 2000, 31, 1029.

31. Cochet, M.; Louam, G.; Quillard, S.; Buisson, J. P.; Lefrant, S.; J. Raman Spectrosc. 2000, 31, 1041.

32. Furukawa, Y.; Ueda, F.; Hyodo, Y.; Harada, I.; Nakajima, T.; Kawagoe, T.; Macromolecules 1998, 21, 1297.

33. Louam, G.; Lapskowski, M.; Quillard, S.; Pron, A.; Buisson, J. P.; Lefrant, S.; J. Phys. Chem. 1996, 100, 6998.

34. Job, A. E.; Constantino, C. J. L.; Mendes, T. S. G.; Teruya, M. Y.; Alves, N.; Mattoso, L. H. C.; J. Spectrosc. 2003, 34, 831.

35. Lin-Vien, D.; Colthup, N. B.; Fateley, W. G.; Grasselli, J. G.; The Handbook of Infrared and Raman Characteristic Frequencies of Organic Molecules, Academic Press: Toronto, 1991.

36. Gonçalves, D.; Mattoso, L. H. C.; Bulhões, L. O. S.; Electrochim. Acta 1994, 39, 2271.

37. Widera, J.; Palys, B.; Bukowska, J.; Jackowska, K.; Synth. Met. 1998, 94 , 265.

38. Widera, J.; Grochala, W.; Jackowska, K.; Bukowska, J.; Synth. Met. 1997, 89, 29.

39. Patil, S.; More, M. A.; Patil, P. P.; J. Appl. Polym. Sci. 1999, 74, 3009.

40. Miras, M. C.; Barbero, C.; Kötz, R.; Haas, O.; J. Electroanal. Chem. 1994, $369,193$. 


\section{NANOCOMPÓSITOS ELETROATIVOS DE POLI-O-METOXIANILINA E POLISSACARÍDEOS NATURAIS}

Carla Eiras, Ionara Nayana Gomes Passos, Ana Cristina Facundo de Brito e José Ribeiro dos Santos Júnior

Departamento de Química, Centro de Ciências Naturais, Universidade Federal do Piauí, Campus Universitário Ministro Petrônio, 64049-550 Teresina - PI, Brasil

Valtencir Zucolotto* e Osvaldo N. Oliveira Jr.

Instituto de Física de São Carlos, Universidade de São Paulo, , CP 369, 13560-970 São Carlos - SP, Brasil.

Igor L. Kitagawa e Carlos J. L. Constantino

Departamento de Física Química e Biologia, Faculdade de Ciências e Tecnologia, Universidade Estadual Paulista, , CP 467, 19060-900, Presidente Prudente - SP, Brasil

Helder Nunes da Cunha

Departamento de Física, Centro de Ciências Naturais, Universidade Federal do Piauí, Campus Universitário Ministro Petrônio Portella, 64049-550 Teresina - PI, Brasil

Na Figura 1S(A) estão os espectros Raman dos filmes de POMA-PVS (50 bicamadas) e de POMA-chichá (20 bicamadas), bem como a imagem óptica (50x) em 1S(B) para o filme POMAchichá com a distribuição em duas dimensões da intensidade da banda $1356 \mathrm{~cm}^{-1}$ ao longo da área destacada. A Figura 1S(C) mostra a distribuição em três dimensões da intensidade da banda $1356 \mathrm{~cm}^{-1}$ ao longo da área destacada em $1 \mathrm{~S}(\mathrm{~B})$. Esta distribuição foi obtida ao longo de uma área de cerca de $40 \mu \mathrm{m}$ x $40 \mu \mathrm{m}$ através do chamado mapeamento Raman (modo "line focus"), sendo a cor branca indicativa de maiores intensidades. Pode-se observar, tanto pela imagem óptica quanto pelo mapeamento Raman ("imagem química"), que o filme POMA-chichá é bastante uniforme morfológica e quimicamente em escala micrométrica (tais medidas foram realizadas em diferentes regiões do filme). Vale destacar que a variação da intensidade da banda $1356 \mathrm{~cm}^{-1}$ observada em $1 \mathrm{~S}(\mathrm{~B})$ e $1 \mathrm{~S}(\mathrm{C})$ é relativamente pequena considerando-se a escala de cores. Além disto, os espectros Raman da POMA-chichá e da POMA-PVS obtidos com laser em $785 \mathrm{~nm}$ são bastante semelhantes, o que indica pelos picos em 577, 1356 e $1597 \mathrm{~cm}^{-1}$ que a POMA permanece no estado de sal de esmeraldina em ambos os filmes, ou seja, a POMA interage com a goma e com o PVS durante a fabricação dos filmes sofrendo uma desdopagem, porém uma vez dopada após o crescimento do filme, assim se mantém.

$\mathrm{Na}$ Figura $2 \mathrm{~S}$ são mostrados dois entre vários espectros Raman

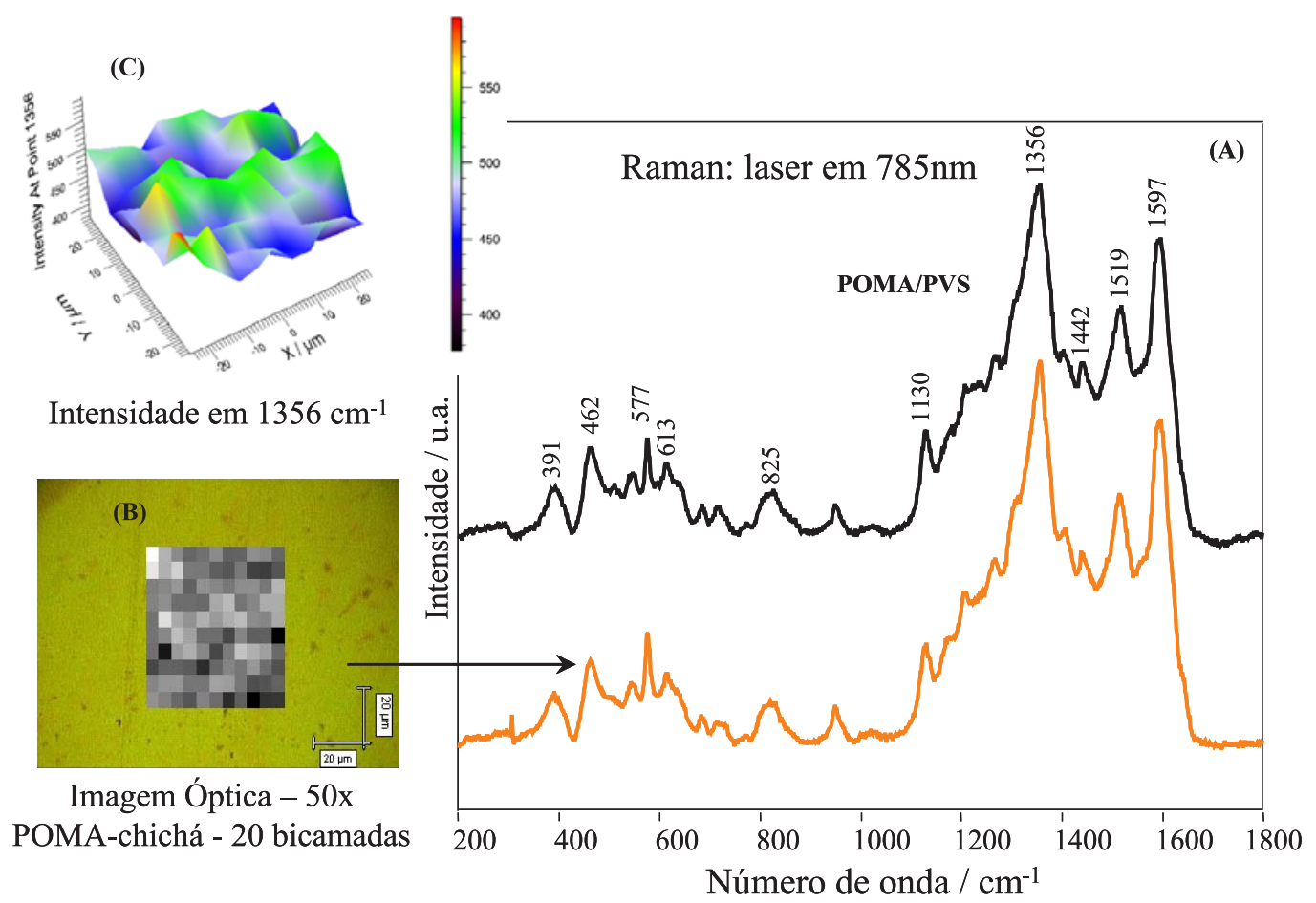

Figura 1S. Espectros Raman dos filmes automontados de POMA-PVS (50 bicamadas) e POMA-chichá (20 bicamadas) (A); imagem óptica para o filme POMAchichá com a distribuição em 2D da intensidade da banda $1356 \mathrm{~cm}^{-1}$ ao longo da área destacada (B); distribuição em $3 D$ da intensidade da banda $1356 \mathrm{~cm}$ ${ }^{I}$ ao longo da área destacada em $(B)(C)$

\footnotetext{
*e-mail: zuco@if.sc.usp.br
} 
de POMA-PVS (50 bicamadas) e de POMA-caraia (20 bicamadas) coletados em diferentes regiões destes filmes, juntamente com as imagens ópticas de ambos os filmes. Novamente pode-se notar a uniformidade morfológica dos dois filmes em escala micrométrica. A semelhança entre os vários espectros obtidos (não mostrados) indica que tal uniformidade, além de morfológica, é química. A goma de caraia também não afeta a dopagem da POMA após a fabricação do filme, a qual se encontra no estado de sal de esmeraldina, evidenciado pelos picos Raman em 577, 1356 e $1597 \mathrm{~cm}^{-1}$. A Figura 3S ilustra os espectros Raman dos filmes de POMA-PVS (50 bicamadas) e de POMA-angico (20 bicamadas) e a imagem óptica do filme POMA-angico. Ao contrário dos filmes POMA-caraia e POMAchichá, as imagens revelam a não-uniformidade morfológica do filme POMA-angico em escala micrométrica, o que contrasta com a uniformidade química evidenciada pela semelhança dos espectros Raman coletados em diferente regiões do filme como mostrado na Figura 3S. Observa-se ainda que os espectros do filme de POMAPVS e de POMA-angico, obtidos com laser de $785 \mathrm{~nm}$, são muito semelhantes e a presença das bandas em 577,1356 e $1597 \mathrm{~cm}^{-1}$ confirma que a POMA permanece no estado de sal de esmeraldina.

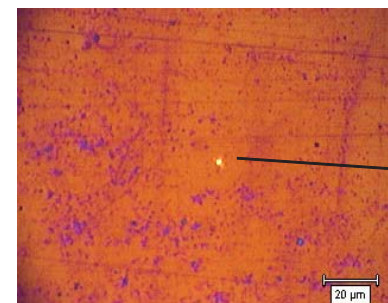

Imagem Óptica - 50x POMA-PVS - 50 bicamadas

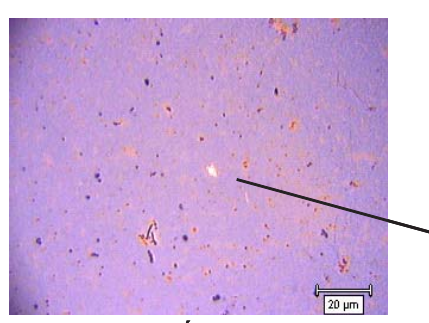

Imagem Óptica $-50 \mathrm{x}$ POMA-caraia - 20 bicamadas

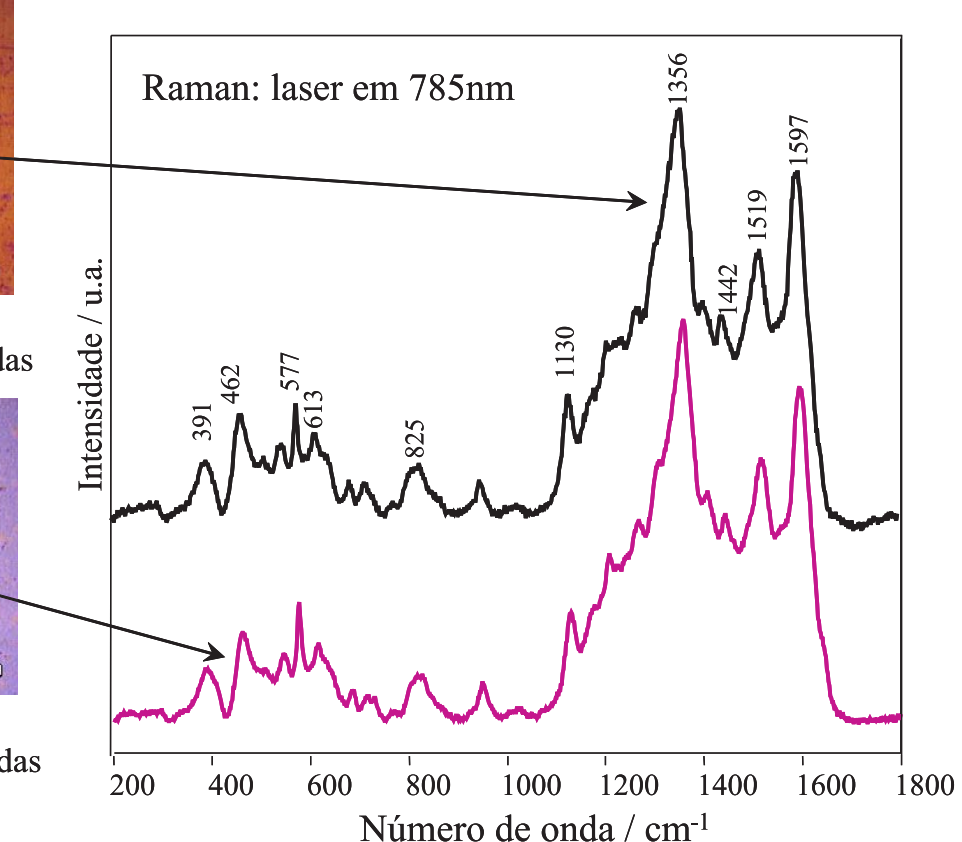

Número de onda $/ \mathrm{cm}^{-1}$

Figura 2S. Espectros Raman dos filmes automontados de POMA-PVS (50 bicamadas) e POMA-caraia (20 bicamadas) com imagens ópticas para ambos os filmes

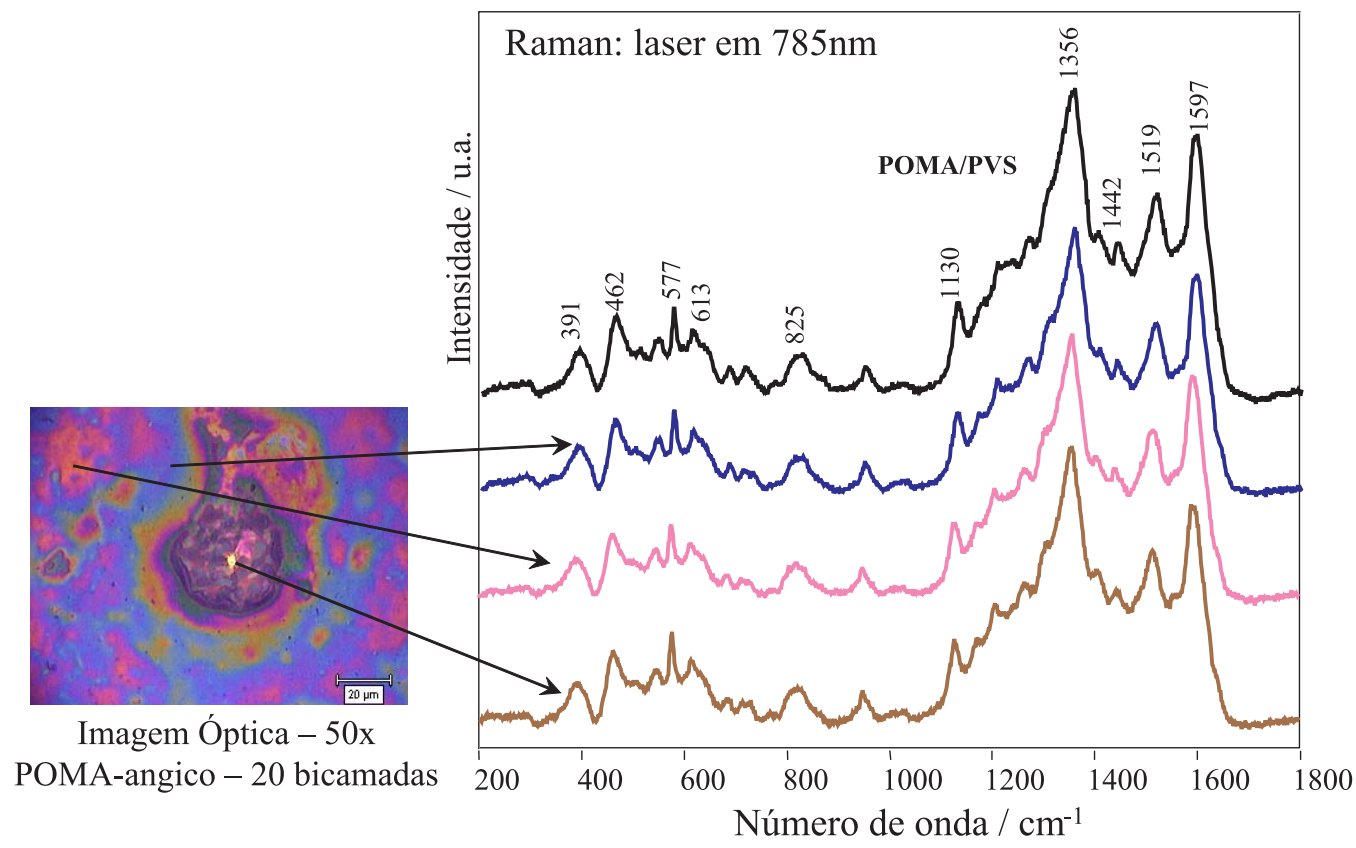

Figura 3S. Espectros Raman dos filmes automontados de POMA-PVS (50 bicamadas) e POMA-angico (20 bicamadas) com imagem óptica do filme POMAangico 